\title{
Estimação do Sobrediagnóstico no Rastreamento Mamográfico: uma Avaliação Crítica
}

doi: https://doi.org/10.32635/2176-9745.RBC.2021v67n2.1281

\author{
Estimation of Overdiagnosis in Mammographic Screening: a Critical Assessment \\ Estimación del Sobrediagnóstico en el Cribado Mamográfico: una Evaluación Crítica
}

Arn Migowski'; Paulo Nadanovsky²; Cid Manso de Mello Vianna ${ }^{3}$

\section{INTRODUÇÃO}

Por sua magnitude e consequências para a saúde, o sobrediagnostico é considerado o dano mais importante associado ao rastreamento mamográfico ${ }^{1}$. Contudo, sua definição ainda é controversa ${ }^{2-4}$ e não há consenso ou consistência sobre a melhor forma de cálculo ${ }^{3,5}$. Essas divergências vêm sendo usadas para desqualificar as estimativas de sobrediagnóstico de forma geral, sem, no entanto, discutir a fundo quais seriam as mais confiáveis ${ }^{1,6}$.

Sobrediagnóstico é o diagnóstico de casos de câncer que nunca iriam se manifestar clinicamente caso não tivessem sido detectados pelo rastreamento ${ }^{2,7}$ ou que náo se exteriorizariam clinicamente em funçâo de causas de morte competitivas ${ }^{7,8}$. Esta última definição ganha relevância em mulheres com comorbidades ou idosas, mas é controversa em períodos de seguimento muito longos ${ }^{5,9}$.

O sobrediagnóstico deve incluir tanto casos de câncer in situ quanto invasivo ${ }^{10}$. A regressão espontânea de carcinoma ductal in situ (CDIS) é relativamente comum e existem situaçôes documentadas do mesmo fenômeno em câncer invasivo, os quais corresponderiam a mais da metade dos casos de sobrediagnóstico ${ }^{10-12}$.

O objetivo do presente artigo é discutir implicaçóes do uso de diferentes desenhos de estudo e formas de cálculo para estimação do sobrediagnóstico de câncer de mama.

\section{DESENVOLVIMENTO}

Embora a existência de sobrediagnóstico no rastreamento mamográfico seja praticamente consensual, sua magnitude ainda é controversa ${ }^{6}$. Um dos motivos para variação é o desenho de estudo, que pode ser ensaios clínicos randomizados (ECR), estudos observacionais ou de modelagem.
As estimativas de tempo de antecipação (TA) baseadas em modelagem variaram de um a sete anos, sugerindo que seria necessário longo seguimento para estimar o sobrediagnóstico. Entretanto, essas modelagens superestimam o TA, por ignorarem o sobrediagnóstico e causas de morte competitivas, assumindo que todos os tumores progridem, variando a velocidade de crescimento $^{13}$. Contudo, o TA real seria de apenas um ano, inferindo-se a partir da diferença de diâmetros dos tumores dos grupos de intervençáo dos ECR de rastreamento ${ }^{14}$. Erros de estimação da duração da fase pré-clínica potencialmente detectável pelo rastreamento (soujorn time) também são causas de variação nas estimativas baseadas em modelagem ${ }^{15}$.

Outra forma de inferir o TA real é a análise de variaçóes da incidência de câncer no rastreamento, pois é esperado que haja uma redução de câncer de intervalo comparada às incidências de base, pela detecção antecipada de muitos casos. Caso o TA fosse longo, como estimado nesses modelos, essa redução duraria muitos anos, mas ela volta aos patamares pré-rastreamento em apenas dois a três anos após a última rodada ${ }^{14}$. A inclusão dos casos de sobrediagnóstico nas estimativas de TA as aumenta artificialmente em até nove anos, dependo da faixa etária.

Se de um lado a inclusão de cânceres detectados anos após o término dos ECR dilui o sobrediagnóstico; de outro, considerar apenas o período de vigência do ECR pode superestimá-lo, analisando como sobrediagnóstico casos de câncer que tiveram detecção antecipada. É preciso definir um tempo máximo para a inclusão de casos, estimando o TA e adicionando esse tempo após o término do período de intervenção do ECR. Um período de seguimento, de três a cinco anos após o término dos ECR, seria mais do que suficiente para estimar o sobrediagnóstico, controlando pelo TA e evitando a

'Instituto Nacional de Cardiologia (INC); Instituto Nacional de Câncer José Alencar Gomes da Silva (INCA). Rio de Janeiro (RJ), Brasil. E-mail: arnmigowski@yahoo.com.br. Orcid iD: https://orcid.org/0000-0002-4861-2319

${ }^{2}$ Instituto de Medicina Social da Universidade do Estado do Rio de Janeiro (IMS/Uerj); Escola Nacional de Saúde Pública da Fundação Oswaldo Cruz (Ensp/Fiocruz). Rio de Janeiro (RJ), Brasil. E-mail: nadanovsky@ims.uerj.br. Orcid iD: https://orcid.org/0000-0003-3345-9873

${ }_{3}^{3}$ IMS/Uerj. Rio de Janeiro (RJ), Brasil. E-mail: cdvianna@gmail.com. Orcid iD: https://orcid.org/0000-0003-0252-1144

Endereço para correspondência: Arn Migowski. Rua das Laranjeiras, 374, $5^{\circ}$ andar - Laranjeiras. Rio de Janeiro (RJ), Brasil. CEP $22240-002$. E-mail: arnmigowski@yahoo.com.br 
diluição pela contaminação do grupo controle (GC) ao longo dos anos ${ }^{14}$.

Uma das evidências da existência de sobrediagnóstico no rastreamento foi o aumento exponencial de câncer localizado após sua introdução, sem que houvesse posterior compensação com diminuição dos casos diagnosticados em estádios avançados ${ }^{11}$.

Estudos ecológicos e de coorte bem conduzidos são considerados boas opçóes para a quantificação de sobrediagnóstico ${ }^{16}$, produzindo estimativas entre $40 \%$ e $60 \%$, embora parte possa ser explicada pelo aumento da incidência associada à popularização da terapia de reposição hormonal nos anos 1990 e pela maior sensibilidade das mamografias digitais quando comparadas às usadas nos $\mathrm{ECR}^{17,18}$.

Um estudo ecológico estimou que $31 \%$ de todos os casos de câncer de mama diagnosticados nos Estados Unidos, durante três décadas em mulheres com 40 anos ou mais, seriam sobrediagnóstico ${ }^{11}$. Essa proporção poderia ter aumentado para $45 \%$ com mudança de denominador, se contabilizados apenas os casos detectados no rastreamento ${ }^{19}$.

No programa de rastreamento holandês, a estimativa, após dedução do TA clínico, é de 14 casos de sobrediagnóstico para cada morte por câncer de mama evitada em mulheres entre 50 e 74 anos, correspondendo a 32\% entre os cânceres detectados naquelas convidadas para rastreamento e $52 \%$ dos cânceres detectados no rastreamento, resultados atribuídos em parte à extensão do rastreamento para além dos 69 anos, descontados casos diagnosticados como in situ ou estádio I que evoluíram para II a IV ${ }^{20}$.

$\mathrm{Na}$ Dinamarca, o rastreamento foi introduzido apenas em parte do país por décadas, criando um GC natural. Nesse país, a estimativa de sobrediagnóstico tende a ser menor pela faixa etária rastreada e baixas taxas de adesão, reconvocação e detecção de CDIS, sendo de 33\%, após ajuste, diminuindo o excesso de casos na população-alvo (50-69) pela queda de incidência ocorrida em mulheres com mais de 70 anos $^{21}$.

A existência de GC concorrente com alocação aleatória da intervenção é uma vantagem dos ECR com relação aos estudos observacionais na estimativa de sobrediagnóstico, uma vez que fornece a linha de base para a comparaçáo do excesso de incidência ${ }^{8}$. A ausência de GC faz com que seja necessário projetar qual seria a incidência na linha de base, usando, por exemplo, séries históricas de grupos etários não rastreados. Usando-se dados de ECR, as estimativas tendem a ser semelhantes se houver similaridade de faixa etária, periodicidade, adesão ao rastreamento, contaminaçáo do GC e forma de cálculo ${ }^{7,22}$.

Periodicidades menores tendem a aumentar o sobrediagnóstico ${ }^{7}$. Menor adesão ao rastreamento no grupo de intervenção tende a subestimá-lo, sendo o $U K$ Age Trial o exemplo mais extremo, com apenas $68 \%$ de adesão $^{22}$.

A contaminação do GC pode ocorrer tanto no período do estudo quanto no seguimento após término, subestimando o sobrediagnóstico. A inclusão de todos os cânceres detectados em longos seguimentos pode diluir as estimativas pela metade ou até mais ${ }^{15}$.

Na revisão sistemática Cochrane, as estimativas de sobrediagnóstico foram de $29 \%$, incluindo todos os ECR, e de 33\%, contabilizando apenas casos detectados antes do rastreamento ser oferecido ao $\mathrm{GC}^{19}$. Todas incluíram a faixa de 40 a 49 anos a qual apresenta mais sobrediagnóstico. Em apenas dois ECR, o rastreamento mamográfico não foi oferecido ao GC após o término: Malmö e Canadian National Breast Screening Study (CNBSS), embora tenham sofrido contaminação durante o período de intervençãa ${ }^{19}$. No Malmö, apenas a um subgrupo do GC entre 55 e 69 anos não foi oferecido rastreamento após o período de intervenção ${ }^{2}$.

Outra fonte de discrepância entre as estimativas de sobrediagnóstico é a forma de cálculo, especialmente no que diz respeito a diferenças de denominador. As opçóes mais usadas como denominador encontram-se no Quadro 1. A forma de cálculo pode gerar discrepâncias facilmente corrigíveis, não representando inconsistência como muitos alegam $^{6}$. Isso fica evidente comparando as estimativas dos ensaios Malmö e CNBSS, usando a mesma fórmula ${ }^{23,24}$.

Um painel independente propôs o uso dos denominadores 2 e 4 (Quadro 1), ambos incluindo CDIS ${ }^{1}$. Outros também defendem o uso de todos os cânceres do grupo rastreado no denominador, pois os ECR tiveram diferentes periodicidades de rastreio, o que influencia a incidência de cânceres de intervalo ${ }^{16}$.

Os ECR são a fonte mais confiável para estimação do sobrediagnóstico ${ }^{25}$. Contudo, tendem a subestimar a magnitude na prática clínica atual, por terem utilizado periodicidades maiores, permitido diversas formas de rastreamento no GC e usado mamografias menos sensíveis ${ }^{5,6}$.

No UK Age Trial, aparentemente há pouco sobrediagnóstico se considerado o período de seguimento após o término do ensaio, no qual o rastreamento mamográfico foi ativamente oferecido ao $\mathrm{GC}^{26}$. Porém, com base na incidência de câncer no GC no período de intervenção, é possível inferir que a proporção de sobrediagnóstico foi de $35 \%{ }^{27}$.

Autores do Malmö estimaram sobrediagnóstico em 10\%, 15 anos após seu término, em mulheres entre 55 e 69 $\operatorname{anos}^{28}$. Esse número está subestimado pela contaminação de $24 \%$ durante o estudo, diminuindo o contraste da incidência entre grupos e, se mantida nesses 15 anos 
Quadro 1. Diferentes numeradores e denominadores usados no cálculo da proporção de sobrediagnóstico nos ensaios clínicos de rastreamento mamográfico

\section{Denominador ${ }^{a}$}

1. Cânceres detectados pelo rastreamento mamográfico durante o período de intervenção

2. Todos os cânceres detectados durante o período de intervenção (incluindo cânceres de intervalo)

3. Todos os cânceres detectados durante o período de intervenção mais um período adicional de seguimento

4. Todos os cânceres detectados durante toda a vida das mulheres a partir da data do início do rastreamento no grupo experimental

5. Cânceres detectados pelo rastreamento mamográfico durante o período de intervenção mais um período adicional de seguimento

6. Cânceres detectados apenas pelo rastreamento mamográfico durante o período de intervenção mais um período adicional de seguimento (não palpáveis)

\section{Numerador}

1. Diferença entre casos de câncer nos grupos de intervenção e controle durante o estudo

2. Diferença entre casos de câncer nos grupos de intervenção e controle no mesmo período de seguimento

(a) todos se referem a casos de câncer de mama no grupo experimental composto por mulheres convidadas para o rastreamento durante o estudo.

após o término, a estimativa subiria para $20 \%$ usando o mesmo cálculo ${ }^{24}$. Analisando apenas casos detectados no grupo experimental durante o estudo, em vez de todos nesses 15 anos, a proporção subiria de 10\% para
15\%. Nesse cálculo, o numerador original foi mantido (número 1 do Quadro), mas o denominador passou a incluir menos casos. Essa estimativa ainda subestima o sobrediagnóstico, pois contabiliza todos os cânceres detectados, incluindo os de intervalo que possuem pior prognóstico. Considerar no denominador apenas cânceres detectados pelo rastreamento diminuiria o denominador e aumentaria o sobrediagnóstico para $24 \%{ }^{29}$. Considerando a contaminação do GC, essa estimativa aumentaria ainda mais. Se usarmos essa mesma forma de cálculo no CNBSS II, ter-se-ia um valor de $37 \%$.

O CNBSS é reconhecido como uma fonte confiável para estimar sobrediagnóstico ${ }^{30,31}$. A forma de cálculo utilizada foi a razão entre o numerador 2 e o denominador 1 do Quadro $^{23}$. Por ter utilizado rastreamento com exame clínico, o CNBSS identificou cânceres detectados exclusivamente pela mamografia. Suas estimativas de sobrediagnóstico podem estar subestimadas por contaminação do GC, que ocorreu em algumas províncias pela implantação de programas de rastreamento, diminuindo as estimativas em cinco anos de seguimento ${ }^{19}$. Estimativas de sobrediagnóstico logo após o término do estudo tendem a superestimá-lo por não considerarem o TA de aproximadamente um ano. As estimativas em três anos após o término são as mais confiáveis, evitando a contaminação em períodos de seguimento maiores (Tabela 1).

\section{CONCLUSÃO}

As melhores evidências disponíveis indicam que 25\% a 30\% dos cânceres detectados no rastreamento são sobrediagnóstico entre 50 e 69 anos, sendo maior do que $40 \%$ entre 40 e 49 anos. Diversos fatores explicam as

Tabela 1. Estimativas de sobrediagnóstico nos ensaios clínicos de Malmö I, CNBSS e UK Age Trial, incluindo cânceres invasivos e in situ ${ }^{a}$

\begin{tabular}{|c|c|c|c|c|}
\hline & $\begin{array}{l}\text { 40-49 anos } \\
\text { CNBSS I I3 }\end{array}$ & $\begin{array}{c}\text { 50-59 anos } \\
\text { CNBSS II }\end{array}$ & $\begin{array}{l}\text { 55-69 anos } \\
\text { Malmö I24 }\end{array}$ & $\begin{array}{l}40-49 \text { anos } \\
\text { UK Age Trial }\end{array}$ \\
\hline Durante o estudo & $37 \%$ & $37 \%$ & - & \\
\hline 1 ano após o estudo & $40 \%$ & $38 \%$ & - & - \\
\hline 2 anos após o estudo & $43 \%$ & $34 \%$ & - & - \\
\hline 3 anos após o estudo & $43 \%$ & $30 \%$ & - & - \\
\hline 4 anos após o estudo & $41 \%$ & $26 \%$ & - & - \\
\hline 5 anos após o estudo & $41 \%$ & $25 \%$ & - & - \\
\hline 10 anos após o estudo & $52 \%$ & $14 \%$ & - & - \\
\hline 15 anos após o estudo & $44 \%$ & $14 \%$ & $24 \%$ & - \\
\hline 20 anos após o estudo & $55 \%$ & $16 \%$ & - & $35 \%{ }^{c}$ \\
\hline 5 anos após o estudo b & $100 \%$ & $44 \%$ & - & - \\
\hline
\end{tabular}

(a) O numerador é a diferença no número de cânceres no braço com mamografia e no grupo controle, já o denominador é formado pelos cânceres detectados no rastreamento no braço com mamografia; (b) Considerados apenas os cânceres detectados pelo rastreamento mamográfico; (c) Considerada a incidência esperada no grupo controle na ausência de rastreamento. 
divergências encontradas na literatura, tais como adesão ao rastreamento, periodicidade, faixa etária, contaminação do GC, TA estimado e diferenças no denominador utilizado. Ao homogeneizar esses fatores, as estimativas de sobrediagnóstico dos principais estudos passam a apresentar consistência.

\section{CONTRIBUIÇÕES}

Arn Migowski contribuiu na concepção e no planejamento do estudo; na obtenção, análise e interpretação dos dados; assim como na redação e revisão crítica com contribuição intelectual. Paulo Nadanovsky e Cid Manso de Mello Vianna contribuíram na interpretação dos dados; assim como na revisão crítica com contribuição intelectual. Todos os autores aprovaram a versão final a ser publicada.

\section{DECLARAÇÃO DE CONFLITO DE INTERESSES}

Nada a declarar.

\section{FONTES DE FINANCIAMENTO}

Não há.

\section{REFERÊNCIAS}

1. Brodersen J, Schwartz LM, Heneghan C, et al. Overdiagnosis: what it is and what it isn't. BMJ Evid Based Med. 2018;23(1):1-3. doi: http://doi.org/10.1136/ ebmed-2017-110886

2. Independent UK Panel on Breast Cancer Screening. The benefits and harms of breast cancer screening: an independent review [Internet]. London: Cancer Research UK; 2012 [cited 2020 Oct 7]. Available from: https:// www.cancerresearchuk.org/sites/default/files/breastscreening-review-exec_0.pdf

3. Migowski A, Stein AT, Ferreira CBT, et al. Guidelines for early detection of breast cancer in Brazil. I - Development methods. Cad Saude Publica. 2018;34(6):e00116317. doi: http://doi.org/10.1590/0102-311X00116317

4. Hofmann B. Back to basics: overdiagnosis is about unwarranted diagnosis. Am J Epidemiol. 2019;188(10):1812-7. doi: http://doi.org/10.1093/aje/ kwz148

5. Davies L, Petitti DB, Martin L, et al. Defining, estimating, and communicating overdiagnosis in cancer screening. Ann Intern Med. 2018;169(1):36-43. doi: http://doi.org/10.7326/M18-0694

6. Pace LE, Keating NL. A systematic assessment of benefits and risks to guide breast cancer screening decisions. JAMA. 2014;311(13):1327-35. doi: http:// doi.org/10.1001/jama.2014.1398
7. Myers ER, Moorman P, Gierisch JM, et al. Benefits and harms of breast cancer screening: a systematic review. JAMA. 2015;314(15):1615-34. doi: http://doi. org/10.1001/jama.2015.13183

8. International Agency for Research on Cancer, World Health Organization. IARC Handbooks of cancer prevention: preamble for screening [Internet]. Lyon: IARC; 2019 [cited 2020 Oct 7]. Available from: http:// handbooks.iarc.fr/docs/HB-Preamble-Screening.pdf

9. Etzioni R, Gulati R, Mallinger L, et al. Influence of study features and methods on overdiagnosis estimates in breast and prostate cancer screening. Ann Intern Med. 2013;158(11):831-8. doi: http://doi.org/10.7326/00034819-158-11-201306040-00008

10. Harris RP. Invited commentary: beyond overdiagnosisdiagnosis without benefit. Am J Epidemiol. 2019;188(10):1818-20. doi: http://doi.org/10.1093/ aje/kwz145

11. Bleyer A, Welch HG. Effect of three decades of screening mammography on breast-cancer incidence. $\mathrm{N}$ Engl J Med. 2012;367(21):1998-2005. doi: http://doi. org/10.1056/NEJMoa1206809

12. Zahl PH, Andersen JM, Maehlen J. Spontaneous regression of cancerous tumors detected by mammography screening. JAMA. 2004;292(21):2579-80. doi: http:// doi.org/10.1001/jama.292.21.2579

13. Welch HG, Gorski DH, Albertsen PC. Trends in metastatic breast and prostate cancer--lessons in cancer dynamics. N Engl J Med. 2015;373(18):1685-7. doi: http://doi.org/10.1056/NEJMp1510443

14. Zahl PH, Jørgensen KJ, Gøtzsche PC. Lead-time models should not be used to estimate overdiagnosis in cancer screening. J Gen Intern Med. 2014;29(9):1283-6. doi: http://doi.org/10.1007/s11606-014-2812-2

15. Zahl PH, Jørgensen KJ, Gøtzsche PC. Overestimated lead times in cancer screening has led to substantial underestimation of overdiagnosis. $\mathrm{Br} \mathrm{J}$ Cancer. 2013;109(7):2014-9. doi: http://doi.org/10.1038/ bjc. 2013.427

16. Carter JL, Coletti RJ, Harris RP. Quantifying and monitoring overdiagnosis in cancer screening: a systematic review of methods. BMJ. 2015;350:g7773. doi: https://doi.org/10.1136/bmj.g7773

17. Gøtzsche PC. On the benefits and harms of screening for breast cancer. Int J Epidemiol. 2004;33(1):56-64. doi: https://doi.org/10.1093/ije/dyh014

18. Zahl PH, Maehlen J. Ramifications of screening for breast cancer: definition of overdiagnosis is confusing in follow-up of Malmö trial. BMJ. 2006;332(7543):727-8. doi: https://doi.org/10.1136/bmj.332.7543.727-b

19. Gøtzsche PC, Jørgensen K. Screening for breast cancer with magrapmmohy. Cochrane Database Syst Rev. 2013;2013(6):CD001877. doi: https://doi. org/10.1002/14651858.CD001877.pub5 
20. Autier P, Boniol M, Koechlin A, et al. Effectiveness of and overdiagnosis from mammography screening in the Netherlands: population based study. BMJ. 2017;359:j5224. doi: https://doi.org/10.1136/bmj. j5224

21. Jørgensen KJ, Zahl PH, Gøtzsche PC. Overdiagnosis in organised mammography screening in Denmark. A comparative study. BMC Womens Health. 2009;9:36. doi: https://doi.org/10.1186/1472-6874-9-36

22. Jacklyn G, Glasziou P, Macaskill P, et al. Meta-analysis of breast cancer mortality benefit and overdiagnosis adjusted for adherence: improving information on the effects of attending screening mammography. Br J Cancer. 2016;114(11):1269-76. doi: https://doi.org/10.1038/ bjc. 2016.90

23. Baines CJ, To T, Miller AB. Revised estimates of overdiagnosis from the Canadian National Breast Screening Study. Prev Med. 2016;90:66-71. doi: https:// doi.org/10.1016/j.ypmed.2016.06.033

24. Gøtzsche PC. Ramifications of screening for breast cancer: overdiagnosis in the Malmö trial was considerably underestimated. BMJ. 2006;332(7543):727. doi: https:// doi.org/10.1136/bmj.332.7543.727-a

25. Lauby-Secretan B, Scoccianti C, Loomis D, et al. Breastcancer screening - viewpoint of the IARC Working Group. N Engl J Med. 2015;372(24):2353-8. doi: https://doi.org/10.1056/NEJMsr1504363

26. Moss SM, Wale C, Smith R, et al. Effect of mammographic screening from age 40 years on breast cancer mortality in the UK Age trial at 17 years' follow-up: a randomised controlled trial. Lancet Oncol. 2015;16(9):1123-32. doi: https://doi.org/10.1016/ S1470-2045(15)00128-X

27. Autier P. Breast cancer: doubtful health benefit of screening from 40 years of age. Nat Rev Clin Oncol. 2015;12(10):570-2. doi: https://doi.org/10.1038/ nrclinonc. 2015.162

28. Zackrisson S, Andersson I, Janzon L, et al. Rate of overdiagnosis of breast cancer 15 years after end of Malmö mammographic screening trial: follow-up study. BMJ. 2006;332(7543):689-92. doi: https://doi.org/10.1136/ bmj.38764.572569.7C

29. Welch HG, Schwartz LM, Woloshin S. Ramifications of screening for breast cancer: 1 in 4 cancers detected by mammography are pseudocancers. BMJ. 2006;332(7543):727. doi: https://doi.org/10.1136/ bmj.332.7543.727

30. Baker SG, Prorok PC, Kramer BS. Lead time and overdiagnosis. J Natl Cancer Inst. 2014;106(12):dju346. doi: https://doi.org/10.1093/jnci/dju346

31. van den Ende C, Oordt-Speets AM, Vroling H, et al. Benefits and harms of breast cancer screening with mammography in women aged $40-49$ years: a systematic review. Int J Cancer. 2017;141(7):1295-1306. doi: https://doi.org/10.1002/ijc.30794

Recebido em 3/11/2020

Aprovado em 4/12/2020 